\title{
Adressage et migration : le mouvement perpétuel des lymphocytes
}

\author{
Dominique Dunon \\ Beat A. Imhof
}

D. Dunon: Cnrs UMR 7622, Université Paris 6-Cnrs, Équipe Adhérence et migration cellulaires BP 24, Bât. C-30, 9, quai SaintBernard, 75230 Paris Cedex 05, France. B.A. Imhof: Département de pathologie expérimentale, Université de Genève, 1, rue Michel Servet, CH-1211 Genève, Suisse.
Le contrôle des mouvements continuels des lymphocytes dans I'organisme contribue de façon significative à l'efficacité du système immunitaire en assurant une surveillance permanente de I'ensemble des tissus et en ciblant les leucocytes aux sites d'invasion microbienne ou antigénique. Les études réalisées depuis 20 ans dans ce domaine ont montré que I'adressage des lymphocytes dans un organe est assuré par une cascade d'étapes impliquant des molécules d'adhérence et de signalisation. L'élucidation de ce processus devrait permettre à terme de moduler le ciblage des cellules effectrices notamment lors de syndromes inflammatoires.

\section{TIRÉS À PART}

D. Dunon.

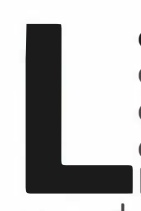

e système immunitaire est constitué d'une large variété de leucocytes et de cellules dendritiques qui surveillent l'apparition d'antigènes étrangers dans les différents tissus de I'individu. Pour établir une réponse immunitaire efficace, l'interaction et la co-localisation des cellules présentatrices des antigènes et des lymphocytes sont des paramètres cruciaux. Dans ce contexte, le fait que les voies de migration des cellules présentatrices des antigènes et des lymphocytes $B$ et T naifs, $n$ 'ayant jamais rencontré I'antigène dont ils sont spécifiques, conduisent aux organes lymphoïdes secondaires, a pour conséquence d'augmenter l'efficacité et la rapidité de la reconnaissance des antigènes. Les organes lymphoïdes secondaires incluent les ganglions lymphatiques périphériques et mésentériques (drainant l'intestin), la rate, et les tissus lymphoïdes associés aux muqueuses (MALT) comprenant notamment les plaques de Peyer, les amygdales, et l'appendice (figure 1). Par ailleurs les lymphocytes, activés en réponse à la reconnaissance de I'antigène, et les lymphocytes mémoires, à nouveau au repos après avoir participé à la réponse immuni- taire, sont dirigés préférentiellement vers les tissus où ils ont une probabilité élevée de rencontrer leur antigène. II faut rappeler que les cellules mémoires sont à la base de la stratégie vaccinale puisqu'elles vont pouvoir persister et protéger l'organisme pendant le reste de sa vie. Ce système très organisé facilitant la reconnaissance des antigènes par les lymphocytes est fondé sur la recirculation continue des lymphocytes entre le sang, les organes lymphoïdes secondaires et les tissus périphériques [1].

\section{Le plan de recirculation des lymphocytes}

La voie classique de migration suivie par les cellules en recirculation est la diapédèse au niveau des veinules postcapillaires dans les tissus périphériques et la plupart des organes lymphoïdes, la migration via les vaisseaux lymphatiques afférents qui conduisent au ganglion lymphatique drainant le tissu, et finalement le passage par les vaisseaux lymphatiques efférents et le canal thoracique qui se déverse dans le sang. Une caractéristique spécifique des organes lymphoïdes secondaires, à l'exception de la rate, est la présence de veinules, 


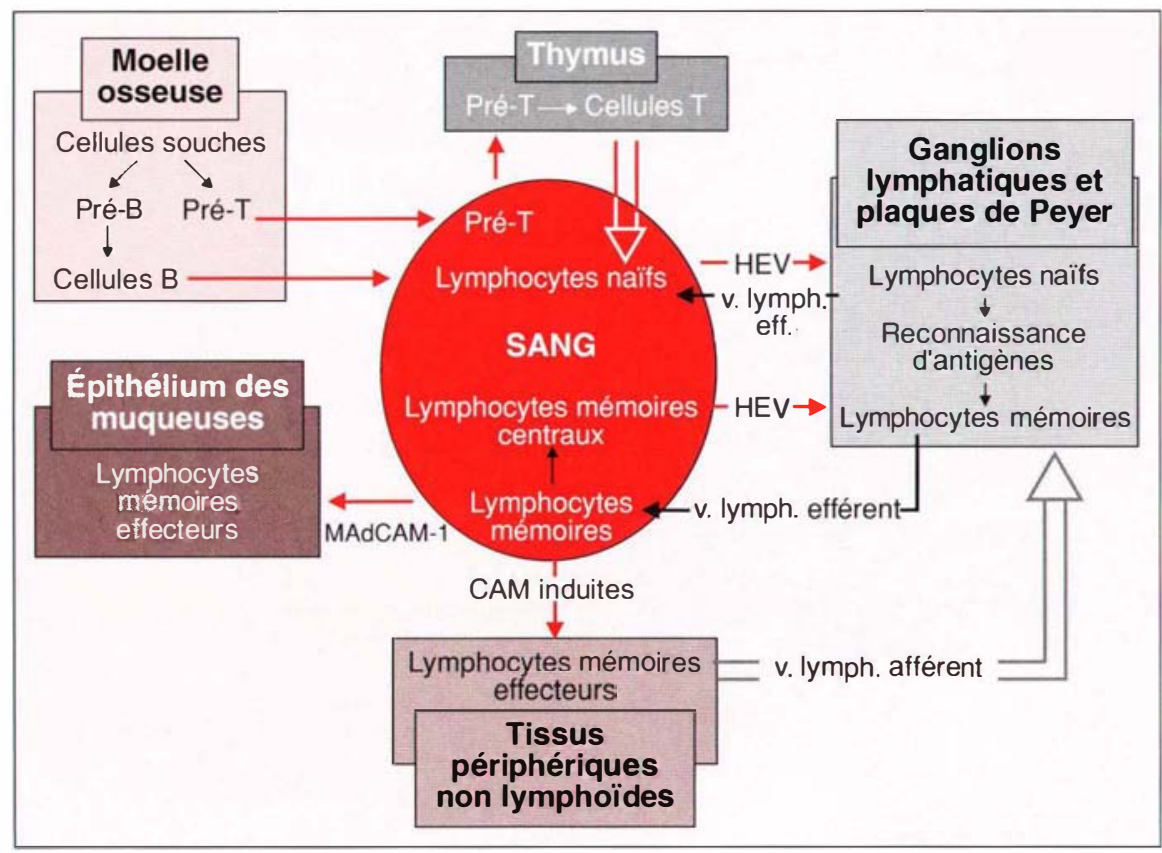

Figure 1. Les patrons de migration et de recirculation des lymphocytes à différents stades de différenciation. Alors que la migration des lymphocytes immatures de la moelle osseuse vers le thymus est seulement unidirectionnelle, les lymphocytes naïs matures recirculent abondamment dans les organes lymphoïdes secondaires, c'est-à-dire ganglions lymphoïdes, plaques de Peyer et rate. II est à noter que la recirculation dans la rate (non figurée) n'implique pas de migration transendothéliale pour des raisons anatomiques. Les cellules naïves et mémoires recirculent dans les ganglions lymphatiques par les HEV, alors que les lymphocytes mémoires effecteurs entrent préférentiellement dans le MALT et l'intestin en utilisant MAdCAM-1 et dans les tissus non lymphoïdes comme la peau en utilisant des molécules d'adhérence cellulaire inductibles.

spécialisées dans la circulation de lymphocytes. Elles sont appelées HEV (pour high endothelial venules) et sont délimitées par des cellules endothéliales présentant une morphologie cuboïdale haute, différente de celle, plate, des cellules endothéliales des autres vaisseaux [2].

Les voies de circulation des lymphocytes ont été principalement étudiées chez le mouton, espèce qui permet la réalisation d'interventions chirurgicales et l'analyse de la circulation lymphocytaire au niveau d'un seul ganglion lymphatique 13]. Les résultats obtenus dans le modèle ovin ont montré que la vaste majorité $(90 \%)$ des lymphocytes recirculants sont des cellules naïves qui entrent dans les ganglions lymphatiques par les HEV alors que seulement $10 \%$ entrent dans le ganglion par les vaisseaux lymphatiques. De plus, les lymphocytes issus d'un tissu donné semblent recirculer préférentiellement vers ce tissu. Ces préférences de domi- ciliation et de recirculation des lymphocytes sont plus importantes pour les tissus lymphoïdes associés au muqueuses (MALT) que pour la peau, et beaucoup moins prononcées pour les autres tissus. La dissection des mécanismes qui contrôlent des voies de migration distinctes a été réalisée dans le modèle murin qui se prête aux expériences in vivo de blocage fonctionnel par des anticorps, et permet la création de modèles génétiques grâce à la technologie de la recombinaison homologue, deux stratégies qui ont permis d'établir les bases moléculaires de l'analyse fonctionnelle de la migration cellulaire (Tableau I).

\section{Le modèle moléculaire de l'interaction lymphocyte-endothélium}

La migration spécifique des lymphocytes vers un tissu donné est fondée sur l'expression différentielle de molécules d'adhérence et de signalisation par les lymphocytes et les cellules endothéliales. En fait, une large variété de molécules, présentant des spécificités partiellement chevauchantes et redondantes, détermine la voie qu'un lymphocyte peut suivre. Un modèle faisant intervenir une cascade de processus d'adhérence entre le leucocyte et l'endothélium a été proposé pour rendre compte des aspects pluriparamètriques de l'extravasation des leucocytes $[4,5]$. La première étape, d'adhérence faible, permet le roulement de la cellule circulante et met en jeu principalement les sélectines. Au cours de cette étape, les chimiokines immobilisées dans le glycocalyx des cellules endothéliales activent les voies de transduction de la cellule circulante, ce qui conduit à l'activation des intégrines qui vont permettre l'arrêt de la cellule circulante à la surface de I'endothélium. Enfin la dernière étape de ce modèle correspond à l'extravasation (ou diapédèse) du leucocyte hors du compartiment vasculaire (figure 2, Tableau (I). La probabilité de la réalisation de chacune de ces étapes dépend de la combinaison de molécules présentes à la surface des deux partenaires. La multiplicité des molécules impliquées et leur redondance partielle ont largement compliqué I'analyse des migrations leucocytaires. Néanmoins, le rôle clef de plusieurs molécules d'adhérence, la sélectine $L$, les intégrines $\alpha 4 \beta 7$ et LFA-1 ( $\alpha \mathrm{L} \beta 2)$, a été clairement démontré.

\section{Les molécules \\ d'adhérence déterminent l'adressage des lymphocytes}

Les voies de migration des cellules naïves sont déterminées par l'expression de la sélectine L

La sélectine L est la molécule responsable du trafic des lymphocytes naïfs vers les ganglions lymphatiques et elle est impliquée dans le recrutement des polynucléaires neutrophiles aux sites d'inflammation [6]. Elle est exprimée par tous les lymphocytes naïfs et par une partie des cellules $T$ mémoires, des neutrophiles, des éosinophiles et des monocytes. La sélectine $\mathrm{L}$ reconnaît des motifs glucidiques présents sur les adressines exprimées par les cellules endothéliales des ganglions lym- 
Tableau I

PHÉNOTYPE DES SOURIS MUTÉES PAR RECOMBINAISON HOMOLOGUE

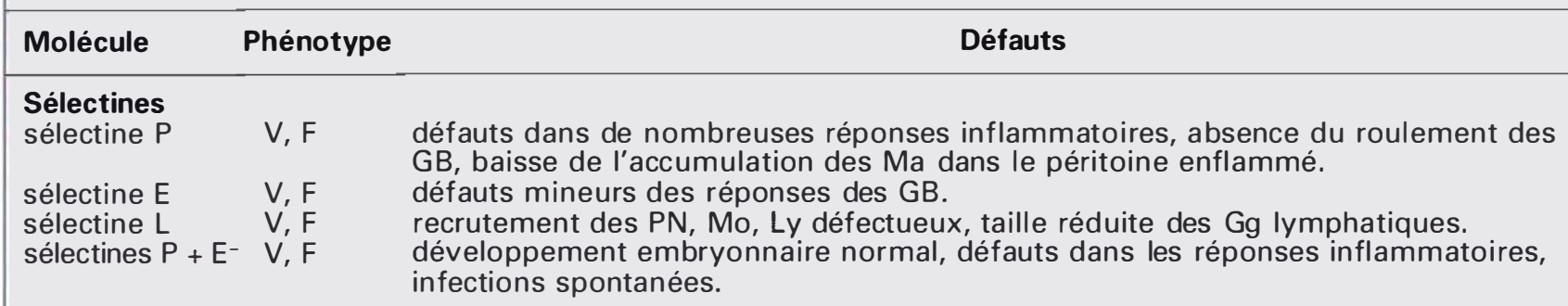

\author{
Ligands des sélectines \\ CD34 V, F \\ baisse \pm du nombre de progéniteurs hématopoïétiques ; \\ accumulation réduite des PNéos lors d'exposition aux allergènes. \\ PSGL-1 V, F retardement de l'infiltration des leucocytes dans des modèles de péritonite \\ GlyCAM-1 V, F hypertrophie des $\mathrm{Gg}$ lymphatiques.
}

Superfamille des immunoglobulines

$\begin{array}{lll}\text { VCAM-1 } & \text { anomalies placenta et cœur ; } 1 \% \text { des souris mutées sont viables et fertiles, } \\ \text { apparemment en bonne santé avec un nombre élevé de Mo. }\end{array}$

ICAM-1 V,F développement embryonnaire normal :

réponses inflammatoires défectueuses: réponse altérée lors de l'hypersensibilité de contact, migration des PN diminuée dans les péritonites induites par des drogues.

ICAM-2 V, F accumulation des PNéos dans l'interstitium pulmonaire;

délai accumulation des PNéos dans les voies aériennes lors d'inflammation allergique du poumon.

PECAM-1/CD31 V,F accumulation des PN entre l'endothélium vasculaire et la membrane basale des microvaisseaux mésentériques enflammés.

Intégrines

$\alpha 4$

$\alpha 9 \quad V$

$\alpha \mathrm{V} \quad \mathrm{P} / \mathrm{E}$

$\alpha L \quad V, F$

$\alpha M \quad V, F$

$\alpha E \quad \quad V$

B1 $\quad E$

B2 $\quad \mathrm{V}, \mathrm{F}$

B3 $\quad \mathrm{V}, \mathrm{F}$

B7 $\quad \mathrm{V}$
E11-14 développement anormal placenta et cœur ;

chimères: blocage de la différenciation des Ly $B$, domiciliation défective des Ly $T$ vers les plaques de Peyer.

mort vers 10 jours après la naissance.

létal pendant l'embryogenèse ou la période périnatale/ développement anormal des vaisseaux cérébraux.

défauts de migration des Ly vers les $\mathrm{Gg}$ périphériques et mésentériques et les sites d'inflammation aiguë.

défauts \pm de la réponse des $\mathrm{PN}$.

réduction du nombre de Ly intra-épithéliaux de l'intestin.

létal avant l'implantation de l'embryon, pas de gastrulation;

chimères: pas de colonisation du foie par les CSH.

résponses inflammatoires anormales: migration anormale des PN vers le péritoine enflammé, retard du rejet de greffes cardiaques.

Sd hémorragique cutané et du tractus digestif: agrégation plaquettaire et rétraction du caillot anormales, allongement temps de saignement.

déficit en Ly du tractus digestif.

\title{
Molécules associées aux intégrines
}

$\begin{array}{lll}\text { IAP } & \text { V,F } & \text { baisse de la résistance aux infections bactériennes et défauts des granulocytes. } \\ \text { CD81 } & \text { V,F } & \text { réponses prolifératives altérées Ly. }\end{array}$

Autres molécules d'adhérence
CD44
CD43
$V, F$
$V, F$
pas de défauts majeurs au cours du développement.
CD38

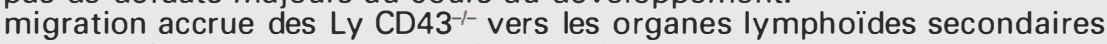 réponses immunitaires humorales altérées.

Chimiokines

SDF-1

$\mathrm{V}, \mathrm{F}$

lymphopoïèse sévèrement réduite, myélopoïèse réduite dans le foie fœtal, absente dans la moelle osseuse. 
Tableau I (suite)

PHÉNOTYPE DES SOURIS MUTÉES PAR RECOMBINAISON HOMOLOGUE

\begin{tabular}{|c|c|c|}
\hline Molécule & Phénotype & Défauts \\
\hline SLC & $\mathrm{V}, \mathrm{F}$ & $\begin{array}{l}\text { mutation plt : baisse de la domiciliation des Ly } T \text { naïfs et des cell dendr stimulées } \\
\text { par l'antigène dans les zones à cellules } T \text { des organes lymphoïdes secondaires. }\end{array}$ \\
\hline Éotaxine-e & $\mathrm{V}, \mathrm{F}$ & pas de défauts majeurs du trafic des Péos. \\
\hline MIP1- $\alpha$ & $\mathrm{V}, \mathrm{F}$ & réponse inflammatoire aux infections virales perturbées. \\
\hline \multicolumn{3}{|c|}{ Récepteurs de chimiokines } \\
\hline CCR1 & $\mathrm{V}, \mathrm{F}$ & défaut de réponse immunitaire : reconnaissance du soi, hématopoïèse, et inflammation. \\
\hline CCR2 & $\mathrm{V}, \mathrm{F}$ & migration anormale des Ma pendant l'inflammation, défaut d'extravasation des Mo. \\
\hline CCR5 & $\mathrm{V}, \mathrm{F}$ & défaut fonctionnel de la fonction des macrophages. \\
\hline CCR7 & $\mathrm{V}, \mathrm{F}$ & $\begin{array}{l}\text { défaut de migration des Ly cell dend vers les Gg lymphatiques, pas de réponse } \\
\text { primaire, réponse humorale retardée. }\end{array}$ \\
\hline CXCR4 & $\mathrm{V}, \mathrm{F}$ & $\begin{array}{l}\text { hématopoïèse et lymphopoïèse anormales dans le foie fœtal et dans la moelle } \\
\text { osseuse. }\end{array}$ \\
\hline CXCR5 & $\mathrm{V}, \mathrm{F}$ & $\begin{array}{l}\text { absence des } \mathrm{Gg} \text { lymphatiques inguinaux, baisse du nombre de plaques de Peyer, } \\
\text { défaut dans la domiciliation des Ly B. }\end{array}$ \\
\hline
\end{tabular}

Les molécules répertoriées ont été impliquées dans l'adhérence et la migration des leucocytes, ou plus spécifiquement dans la recirculation des lymphocytes. En raison des fonctions et des spécificités chevauchantes, l'absence d'une molécule peut souvent provoquer des défauts partiels (par exemple les sélectines $E$ et $P$ ) ou être complètement compensée par d'autres molécules (CD44 et CD38). V: viable; F: fertile; E11-14: létalité embryonnaire au jour 11-14; $P$ : létalité périnatale.

GB: leucocytes, globules blancs; PNéos: polynucléaires éosinophiles; Ma: macrophages; Mo: monocytes; Ly: Iymphocytes; Gg: ganglions; cell dend: cellules dendritiques.

phatiques périphériques (GlyCAM-1Gly cell adhesion molecule -, CD34, et MAdCAM-1 - mucosal addressin cell adhesion molecule-1-) et qui sont fixés à ces protéines par des glycosyltransférases spécifiques des HEV puis modifiés par sulfatation $[7,8]$. Le rôle de la sélectine $L$ est de ralentir la cellule ce qui permet aux étapes subséquentes de se réaliser. Le fait que les cellules $T$ mémoires, qui n'expriment pas la sélectine $L$, entrent avec la même efficacité que les cellules naïves dans les ganglions périphériques via les HEV [9] indique que des voies alternatives peuvent se substituer à la sélectine $L$; dans ce cas précis l'étape de roulement serait assurée par une interaction entre l'intégrine $\alpha 4 \beta 1$ et son ligand endothélial VCAM-1 (vascular cell adhesion molecule) [10].

\section{L'intégrine $\alpha 4 \beta 7$ joue un rôle majeur dans l'adressage des lymphocytes vers l'intestin et les tissus lymphoïdes associés aux muqueuses}

L'intégrine $\alpha 4 \beta 7$ est la molécule qui détermine la domiciliation des lymphocytes vers le MALT et la lamina propria de l'intestin, les ganglions lymphatiques intestinaux et les glandes épithéliales exocrines. Son ligand est la molécule MAdCAM-1, une molécule transmembranaire constituée de deux domaines d'immunoglobuline et d'un domaine mucine très glycosylé. MAdCAM-1 est un excellent exemple de la spécificité tissulaire d'une adressine puisque son expression est restreinte aux HEV du MALT et des veinules de la lamina propria et de la glande mammaire [11]. Bien que la fonction majeure de MAdCAM-1 s'exerce via son interaction avec l'intégrine $\alpha 4 \beta 7$, dans certains tissus elle peut également lier la sélectine $L$ par l'intermédiaire des structures glucidiques de son domaine mucine. La sélectine L et l'intégrine $\alpha 4 \beta 7$ étant toutes deux utilisées par les lymphocytes naîfs qui entrent dans les ganglions mésentériques et les plaques de Peyer, le blocage de l'activité de l'une ou de l'autre des molécules a peu d'effet sur leur migration. En revanche, pour les cellules mémoires qui n'expriment pas la sélectine $L$, l'intégrine $\alpha 4 \beta 7$ joue un rôle décisif pour l'entrée de ces cellules dans le MALT [9].
Un argument supplémentaire en faveur de l'importance de l'intégrine $\alpha 4 \beta 7$ dans la domiciliation des lymphocytes vers le MALT et l'intestin est apporté par l'analyse de la distribution lymphocytaire chez les souris déficientes pour la sous-unité $\beta 7$ des intégrines: en raison du défaut d'immigration des lymphocytes dans la lamina propria, les plaques de Peyer et l'épithélium intestinal, le nombre de lymphocytes est sévèrement réduit dans ces tissus [12]. Cette molécule est importante dans la mise en place de la réponse immunitaire puisque l'élimination de rotavirus de souris immunodéficiente est obtenu par le transfert de cellules T CD8 provenant de souris sauvages infectées par ce même virus, cellules qui expriment $\alpha 4 \beta 7$. En effet, les cellules CD8 négatives pour $\alpha 4 \beta 7$ en sont incapables [13].

\section{Adressage des lymphocytes vers la peau}

Chez I'homme, I'expression de CLA (cutaneous lymphocyte-associated antigen) à la surface des lymphocytes T mémoires a été corrélée avec la capacité de ces cellules de migrer 


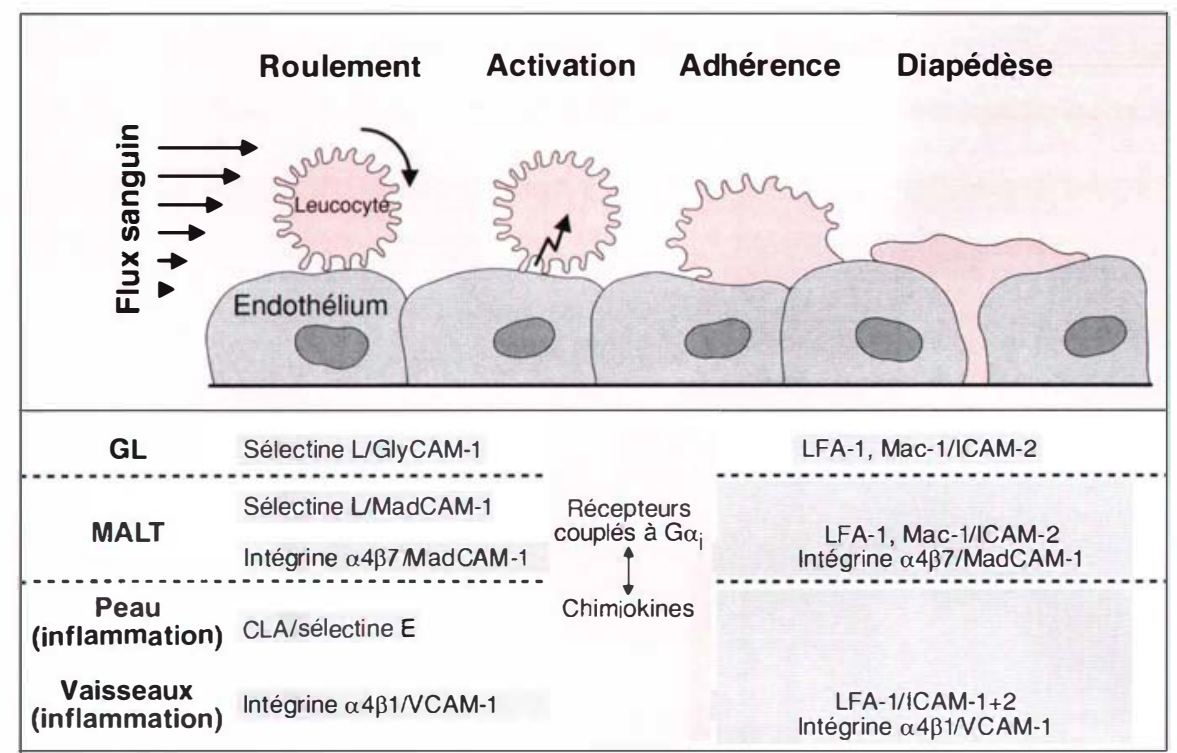

Figure 2. Le modèle de cascade d'adhérence intervenant dans l'émigration des leucocytes du sang. Dans la première étape, les molécules d'adhérence cellulaires interagissent de façon lâche avec leurs ligands exprimés à la surface de la cellule endothéliale, permettant le roulement et le ralentissement des leucocytes circulants. Cette étape permet aux chimiokines présentées par le glycocalix de l'endothélium d'interagir avec leurs récepteurs leucocytaires, ce qui va entrainer l'activation des intégrines exprimées par la cellule circulante. Les intégrines activées interagissent alors avec leurs ligands sur les cellules endothéliales, arrêtent le leucocyte et participent à la diapédèse. Dans ce processus séquentiel, chaque étape dépend de l'étape précédente, en commençant par l'adhérence lâche qui induit le roulement et ainsi de suite. Ainsi, seule une combinaison distincte de récepteurs de chimiokines et de molécules d'adhérence permet au leucocyte d'adhérer et de traverser l'endothélium à un site donné. Inversement, l'endothélium peut sélectivement recruter des sous-populations de leucocytes en présentant les combinaisons complémentaires de molécules d'adhérence et de chimiokines. Les cases sous le schéma indiquent les molécules d'adhérence impliquées dans chaque étape (leucocyte/endothélium) dans différents tissus (ganglions lymphatiques, GL; tissus lymphoïdes associés aux muqueuses, MALT; peau enflammée et sites généraux d'inflammation). Comme l'indiquent les connexions entre les cases, les intégrines $\alpha 4 \beta 1$ et $\alpha 4 \beta 7$ sont impliquées dans plusieurs étapes. II est à noter qu'aux sites d'inflammation, les cytokines $d^{\prime}$ alarme telles que I'IL-1, le TNF ou l'interféron- $\gamma$ produites par les macrophages stimulés par des micro-organismes ou par des lymphocytes $T$ activés par l'interaction avec l'antigène dont ils sont spécifiques, induisent massivement l'expression des molécules d'adhérence par l'endothélium vasculaire. Les molécules $d^{\prime}$ adhérence réglées incluent les sélectines $P$ et $E$ ainsi que les molécules ICAM-1 et VCAM-1.

dans la peau [14]. CLA est un épitope glucidique lié à la protéine PSGL-1 (P-selectin glycoprotein ligand 1) par la fucosyltransférase VII [15]. L'épitope CLA assure la liaison de la cellule circulante à la sélectine $\mathrm{E}$ exprimée par les cellules endothéliales de la peau au cours du processus inflammatoire, et induit le recrutement des cellules T mémoires vers cet organe [16]. en LFA-1 présentent des nombres très faibles de lymphocytes dans les ganglions périphériques mais pas dans la rate [18]. LFA-1, qui a pour ligands les molécules ICAM-(intercellular cell adhesion molecule) 1 et 2 exprimées par les cellules endothéliales, prend place en aval du roulement contrôlé par la sélectine $L$ et ne peut intervenir en son absence |19]. L'analyse de ces mêmes souris $\mathrm{LFA}^{-/}$suggère que la domiciliation résiduelle des lymphocytes vers les ganglions semble dépendre des intégrines $\alpha 4 \beta 1$ et $\alpha 4 \beta 7$ [20] bien que, dans des conditions non inflammatoires, l'expression de VCAM-1, le ligand de ces intégrines, à la surface des HEV soit controversée. II est à noter que les interactions des intégrines $\alpha 4 \beta 1$ et $\alpha 4 \beta 7$ avec VCAM- 1 , voire de $\alpha 4 \beta 7$ et MAdCAM-1, jouent un double rôle en pouvant aussi bien assurer le roulement que l'adhérence forte en I'absence soit de la sélectine $L$ soit de LFA-1.

\section{Les chimiokines indiquent le chemin}

Les intégrines sont des éléments importants des migrations cellulaires et de la recirculation qui déterminent les routes potentielles qu'une cellule peut prendre. Néanmoins la capacité d'utiliser ces intégrines est contrôlée par l'expression des récepteurs de chimiokines (Tableau III) qui, lors de leur liaison avec leur ligand, transduisent un signal qui active les intégrines disponibles. En effet, à la surface des lymphocytes circulants, LFA-1 est exprimée dans une conformation de faible affinité pour ses ligands, les molécules ICAMs, et ne peut assurer I'adhérence forte à I'endothélium. Le roulement des cellules sur l'endothélium favorise le contact entre les chimiokines présentées par celui-ci et leurs récepteurs présents à la surface du lymphocyte, permettant à la cellule de collecter les informations spécifiques de l'endothélium concerné. L'interaction d'une chimiokine avec son récepteur active la voie de signalisation dépendant des protéines $G \alpha i$ et qui, elle-même, active les intégrines, notamment LFA- 1 et $\alpha 4 \beta 1$. Ce changement de conformation permet une liaison de forte affinité au ligand et stabilise I'adhérence forte des leucocytes [22]. Un nombre de plus en 
Tableau II

LES MOLÉCULES D'ADHÉRENCE ET LEURS FONCTIONS

\begin{tabular}{|c|c|c|c|c|}
\hline $\begin{array}{l}\text { Récepteur } \\
\text { cellulaire }\end{array}$ & Expression & Ligand & Étape d'Adh & Fonction \\
\hline \multicolumn{5}{|l|}{ Intégrines } \\
\hline $\begin{array}{l}\text { LFA-1 }(\alpha \mathrm{L} \beta 2, \\
\text { CD11a/CD18) }\end{array}$ & GB & ICAM-1,2,3 & Adh forte & $\begin{array}{l}\text { rôle général dans } \\
\text { l'extravasation des GB }\end{array}$ \\
\hline$\alpha 4 \beta 1$ (VLA-4) & Ly & VCAM-1, Fn & $\begin{array}{l}\text { roulement et } \\
\text { Adh forte }\end{array}$ & $\begin{array}{l}\text { adressage des GB aux sites } \\
\text { d'inflammation }\end{array}$ \\
\hline$\alpha 4 \beta 7$ & Ly & $\begin{array}{l}\text { MadCAM-1, Fn, } \\
\text { VCAM-1 }\end{array}$ & $\begin{array}{l}\text { roulement et } \\
\text { Adh forte }\end{array}$ & adressage des Ly vers le MALT \\
\hline $\begin{array}{l}\alpha \mathrm{M} \beta 2 \text { (Mac-1, } \\
\text { CD11b/CD18) }\end{array}$ & Sous-pop de Ly & $\begin{array}{l}\text { ICAM-1, } \\
\text { fibrinogène, iC3b }\end{array}$ & Adh forte & $\begin{array}{l}\text { adressage des GB aux sites } \\
\text { d'inflammation }\end{array}$ \\
\hline$\alpha 9 \beta 1$ & $\begin{array}{l}\text { PN, muscle lisse } \\
\text { et cellules } \\
\text { endothéliales }\end{array}$ & $\begin{array}{l}\text { VCAM-1, } \\
\text { ténascine-C }\end{array}$ & Adh forte & $\begin{array}{l}\text { adressage des PN aux sites } \\
\text { d'inflammation }\end{array}$ \\
\hline \multicolumn{5}{|l|}{ Sélectines } \\
\hline $\begin{array}{l}\text { sélectine L } \\
\text { (MEL-14, } \\
\text { CD62L) }\end{array}$ & GB non activés & $\begin{array}{l}\text { sLex sur } \\
\text { GlyCAM, CD34 } \\
\text { et MadCAM-1 }\end{array}$ & roulement & $\begin{array}{l}\text { adressage des Ly vers les } \\
\text { ganglions lymphatiques, } \\
\text { adressage des GB aux sites } \\
\text { d'inflammation }\end{array}$ \\
\hline $\begin{array}{l}\text { sélectine } \mathbf{P} \\
\text { (CD62) }\end{array}$ & $\begin{array}{l}\text { PI activées, } \\
\text { endothélium } \\
\text { inflammatoire }\end{array}$ & PSGL-1 & roulement & $\begin{array}{l}\text { recrutement des GB aux sites } \\
\text { d'inflammation }\end{array}$ \\
\hline $\begin{array}{l}\text { sélectine E } \\
\text { (CD62E) }\end{array}$ & $\begin{array}{l}\text { endothélium } \\
\text { inflammatoire }\end{array}$ & $\begin{array}{l}\text { CLA, PSGL-1, } \\
\text { ESL-1 }\end{array}$ & roulement & $\begin{array}{l}\text { recrutement des GB aux sites } \\
\text { d'inflammation }\end{array}$ \\
\hline \multicolumn{5}{|l|}{ Superfamille Ig } \\
\hline ICAM-1 & $\begin{array}{l}\text { endothélium } \\
\text { inflammatoire, } \\
\text { Ly activés }\end{array}$ & LFA-1, $\alpha \mathrm{M} \beta 2$ & Adh forte & $\begin{array}{l}\text { recrutement des GB aux sites } \\
\text { d'inflammation }\end{array}$ \\
\hline ICAM-2 & $\begin{array}{l}\text { endothélium et Ly } \\
\text { quiescents }\end{array}$ & LFA-1 & Adh forte & $\begin{array}{l}\text { rôle général dans l'extravasation } \\
\text { des GB }\end{array}$ \\
\hline VCAM-1 & $\begin{array}{l}\text { endothélium } \\
\text { inflammatoire }\end{array}$ & $\alpha 4 \beta 1, \alpha 4 \beta 1$ & Adh forte & $\begin{array}{l}\text { recrutement des GB aux sites } \\
\text { d'inflammation }\end{array}$ \\
\hline MAdCAM-1 & $\begin{array}{l}\text { endothélium } \\
\text { muqueux }\end{array}$ & $\begin{array}{l}\text { Sélectine L, } \\
\alpha 4 \beta 7\end{array}$ & $\begin{array}{l}\text { roulement et } \\
\text { Adh forte }\end{array}$ & $\begin{array}{l}\text { recrutement des Ly vers le } \\
\text { MALT et l'intestin }\end{array}$ \\
\hline \multicolumn{5}{|c|}{ Ligands des sélectines } \\
\hline GlyCAM & HEVs & sélectine $\mathrm{L}$ & roulement & $\begin{array}{l}\text { recrutement des Ly naïs vers } \\
\text { les ganglions Iymphatiques }\end{array}$ \\
\hline CD34 & endothélium & sélectine L & roulement & $\begin{array}{l}\text { recrutement des Ly naïfs vers } \\
\text { les ganglions Iymphatiques et } \\
\text { les sites d'inflammation }\end{array}$ \\
\hline CLA & Ly activés & sélectine $E$ & roulement & $\begin{array}{l}\text { adressage des Ly vers la peau } \\
\text { enflammée }\end{array}$ \\
\hline MAdCAM-1 & $\begin{array}{l}\text { endothélium } \\
\text { des muqueuses }\end{array}$ & sélectine $\mathrm{L}$ & roulement & $\begin{array}{l}\text { recrutement des Ly vers le } \\
\text { MALT }\end{array}$ \\
\hline PSGL-1 & $\begin{array}{l}\text { cellules My, Ly et } \\
\text { cell dend }\end{array}$ & $\begin{array}{l}\text { sélectine } P \text {, } \\
\text { sélectine } E\end{array}$ & roulement & $\begin{array}{l}\text { adressage des GB vers les sites } \\
\text { d'inflammation }\end{array}$ \\
\hline ESL-1 & cellules myéloïdes & sélectine $E$ & roulement & $\begin{array}{l}\text { adressage des GB vers les sites } \\
\text { d'inflammation }\end{array}$ \\
\hline
\end{tabular}

Adh: adhérence; HEV : high endothelial veinules; ICAM : intercellular CAM- ; VCAM : vascular CAM-1;GlyCAM : glycosylation dependent CAM ; CLA : cutaneous lymphocyte antigen, structure glucidique sur PSGL-1; MAdCAM : mucosal addressin CAM ; PSGL-1 : P-selectin glycoprotein ligand ; ESL-1 : E-selectin ligand-1. Abréviations des cellules: voir Tableau I. 
plus important de chimiokines sont impliquées dans le recrutement sélectif de différentes sous-populations de leucocytes des différents tissus. L'une d'elle, la chimiokine SLC (second lymphoid organ chimiokine) (6C) est exprimée par les HEV et les cellules non lymphoïdes des régions riches en cellules $T$ des ganglions lymphatiques de la rate et des plaques de Peyer [ 1 , 23|. La chimiokine SLC induit (1) une rapide activation de l'intégrine LFA-1 et l'arrêt de lymphocytes en roulement in vitro ainsi que (2) le roulement et l'arrêt des cellules $T$ et B naïves $\alpha 4 \beta 7^{+}$sur MAdCAM-1. Enfin, on observe une réduction majeure du nombre de lymphocytes traversant les HEV chez les souris déficientes pour SLC. SLC est la première chimiokine spécifique des cellules $T$ naïves, mais $\mathrm{d}^{\prime}$ autres chimiokines sont spécifiques $d^{\prime}$ autres sous-populations [1]: MIP3 $\alpha$ (macrophage inflammatory protein-3 alpha) agit sur les cellules T CD4 mémoires et pas sur les cellules $\mathrm{T}$ CD4 naïves, alors que SDF (stro- mal-derived factor) et MIP3 3 activent I'adhérence de la plupart des lymphocytes sur ICAM-1 [24]. Une complexité supplémentaire vient du fait qu'une cellule endothéliale peut présenter des chimiokines qu'elle n'a pas synthétisées. En effet, il est vraisemblable que les cellules endothéliales transportent, vers la lumière du vaisseau, par un mécanisme de transcytose, les chimiokines synthétisées par le tissu sous-jacent afin de les présenter aux leucocytes circulants [25]. Cette présentation fait intervenir un domaine liant l'héparine qui immobilise les chimiokines solubles sur les protéoglycanes endothéliaux [26].

L'expression, par les leucocytes, de récepteurs appropriés, conditionne leur réponse aux chimiokines. Le patron d'expression de ces récepteurs non seulement distingue les souspopulations majeures de leucocytes mais est la clé de la spécificité de migration des cellules $T$ auxiliaires (Th1, Th2) et de diverses populations de cellules $T$ mémoires $[21,27]$. Les cellules Th1 et Th2 expriment principalement CXCR3 et CCR5 alors que la migration des cellules $T$ mémoires cutanées $\left(\mathrm{CLA}^{+} \mathrm{CD}^{+}\right)$vers la peau, lorsqu'elle est de siège d'un processus inflammatoire, requiert une interaction entre la chimiokine TARC (thymus and activation-regulated chemokine) et le récepteur CCR4 [28]. En l'absence de CCR7, le récepteur de SLC, la migration des cellules dendritiques et celle des cellules $T$ naïves et $d$ 'une partie des cellules $T$ mémoires vers les ganglions lymphatiques et les plaques de Peyer sont considérablement réduites, ce qui entraîne l'absence de réponse primaire des cellules $T$ et une réponse humorale retardée [29]. L'expression de CCR7 distingue deux populations de cellules $T$ mémoires: les cellules $\mathrm{CCR}^{+}$, ou cellules mémoires centrales, migrent comme les cellules naïves vers les organes lymphoïdes secondaires, alors que les cellules $T$ CCR7-, ou cellules T mémoires effectrices, peuvent entrer dans les tissus

Tableau III

LES CHIMIOKINES ET LEURS RÉCEPTEURS

\begin{tabular}{lll|}
\hline Récepteur & Ligands & Profil d'expression \\
\hline CXCR1 & IL-8, GCP-2 & PN \\
CXCR2 & IL-8, Gro- $\alpha,-\beta,-y$, & PN \\
& NAP-2, ENA-78 & \\
CXCR3 & IP-10, MIG, I-TAC & Ly Th-1 activées, Ly NK \\
CXCR4 & SDF-1 (PBSF) & Mo, cell dend, Ly T et B quiescents \\
CXCR5 (BLR-1) & BCA-1 (BLC) & Ly B \\
CCR1 & MIP-1 $\alpha$, RANTES, & PNéos, Mo, Ly T activés, \\
& MCP-3, -4, MIP-5 & cell dend \\
CCR2 & MCP-1, -2, -3,-4, -5 & PN baso, Mo, Ly T activés, Ly NK \\
CCR3 & Éotaxine-1, RANTES, MIP-5, & PNéos, PN baso, \\
CCR4 & MCP-2,-3,-4, éotaxine-2 & Ly Th2 activés \\
CCR5 & TARC, MDC & Mo, cell dend, Ly Th2 activés \\
CCR6 & MIP-1 $\alpha,-\beta$, RANTES & Ly Th-1 activés, Mo, Ly NK, cell dend \\
CCR7 & EXODUS-1 (LARC, MIP-3 $\alpha)$ & Cell dend matures \\
& EXODUS-3 (ELC, MIP-3 3$)$ & Ly T activés et quiescents \\
CCR8 & EXODUS-2 (SLC, 6Ckine) & \\
CCR9 & I-309 & Mo \\
CCR10 & TECK & thymocytes, Ly T du MALT \\
CX3CR1 & MCP-1, -3, -4, RANTES & placenta, foie fœtal \\
\end{tabular}

$B C A$ : B cell antigen ; $B L C$ : B lymphocyte chemoattractant ; SDF : stromal-derived factor ; PBSF : proB cell stimulating factor ; Gro : growth-related oncogene ; Mig: monokine induced by interferon-gamma ; IP-10: interferon-gamma inducible protein $10 ;$ MIP: macrophage inflammatory protein TARC : thymus and activation-regulated chemokine ; ELC : Epstein Barr virus-induced molecule 1 ligand chemokine; SLC : secondary lymphoid tissue chemokine. TECK : thymus-expressed chemokine: LARC: liver and activation-regulated chemokine; RANTES: regulated upon activation normal T cell expressed and secreted ; MCP: monocyte chemotactic protein-1. Une nouvelle classification des chimiokines et de leurs récepteurs vient d'être proposée [21]. 
périphériques pour participer aux réactions inflammatoires et cytotoxiques. Intégrines et récepteurs de chimiokines contrôlent la spécificité de migration des cellules CCR7 ${ }^{-}$: l'expression de CLA, de l'intégrine $\alpha 4 \beta 1$ et de CCR4 dirige les cellules vers la peau, et celle de $\alpha 4 \beta 7$ et CCR5 les dirigent vers la muqueuse intestinale. Enfin, I'altération de la formation des organes lymphoïdes secondaires observée chez les souris déficientes en $B R L-1$, un récepteur de chimiokine, serait liée à des perturbations de la migration des lymphocytes B. Chez ces souris, les lymphocytes B migrent normalement vers les organes lymphoïdes, mais à l'intérieur de ces organes ces cellules semblent incapables de rejoindre les follicules lymphocytaires [30]. La migration des lymphocytes et leur recirculation dépendent non seulement de la combinaison des molécules d'adhérence mais est aussi déterminée par l'expression des récepteurs de chimiokine appropriés qui vont induire I'activation des intégrines, l'adhérence forte des lymphocytes et leur diapédèse.

\section{Les inconnues de la recirculation des lymphocytes}

L'origine des lymphocytes dont on analyse les migrations est cruciale puisqu'on sait que, dans leur majorité, les lymphocytes sanguins ne recirculent pas. Soit les lymphocytes fraichement retournés dans la circulation sanguine ont besoin de temps pour redevenir compétents pour la migration, soit les cellules qui recirculent très efficacement ne subsistent qu'un temps très court dans la circulation sanguine. Ceci suggère que les lymphocytes sanguins sont de piètres migrateurs qui s'accumulent dans la circulation. De toute façon, seuls $2 \%$ des lymphocytes totaux circulent dans le sang alors que la majeure partie $(98 \%)$ est localisée dans les organes lymphoïdes [31]. Les mécanismes qui déterminent le nombre de lymphocyte dans le sang ne sont pas connus. Des changements apparemment mineurs dans le taux d'entrée, de transit et de sortie des lymphocytes à partir du ou vers le sang, pourraient donc avoir des effets importants sur le nombre de cellules présentes dans la circulation sanguine. Il a ainsi été proposé que la perte des lymphocytes CD4 sanguins pendant les infections par le HIV et le SIV pourrait refléter une altération du trafic des lymphocytes plutôt que traduire leur élimination directe [32, 33].

L'efficacité de la domiciliation d'un lymphocyte vers un organe distinct est vraisemblablement corrélée à sa probabilité d'interagir avec les molécules d'adhérence de l'endothélium. Puisque la rate est l'organe lymphoïde dans lequel le flux sanguin est le plus important, la probabilité d'interaction des lymphocytes avec des molécules d'adhérence y est plus grande que dans les plaques de Peyer, par exemple. Ceci pourrait conduire à une rétention préférentielle des lymphocytes qui ne sont pas rapidement recrutés par les autres organes avec une forte affinité, conférant à la rate un rôle de filtre pour les lymphocytes à faible capacité de recirculation.

La domiciliation et la recirculation des lymphocytes sont des processus dynamiques et l'analyse in vivo, fondée sur l'isolement des lymphocytes, leur marquage et leur ré-injection, interfère généralement avec l'homéostasie du système immunitaire, ne permettant d'appréhender qu'une partie des mécanismes contrôlant les flux de lymphocytes. Des techniques plus raffinées aideront à pister les cellules plus efficacement pendant des périodes beaucoup plus longues. Par exemple, l'activation de gènes marqueurs induits lors des événements de recombinaison génétique devrait se révèler une stratégie très puissante dans un futur proche [34]. En effet, cette approche a déjà permis de marquer spécifiquement des populations lymphocytaires, telles que les cellules mémoires, et de suivre leur devenir lors de transferts adoptifs. Enfin, I'intérêt d'établir et de valider le concept de migrations ordonnées et bien définies réside dans I'utilisation clinique de la compréhension de la réponse immunitaire. En particulier, I'inflammation qui se traduit par une surexpression des molécules d'adhérence impliquées dans la recirculation des lymphocytes et leur migration massive dans ces sites est un domaine d'application privilégié

\section{RÉFÉRENCES}

1. Foussat A, Galanaud P, Émilie D. Les chimiokines et la longue marche des leucocytes. Med Sci 2000; 16:757-66.

2. Kraal G, Mebius, RE. High endothelia venules: lymphocyte traffic control and controlled traffic. Adv Immunol 1997; 65: 347-95.

3. Young AJ. The physiology of lymphocyte migration through the single lymph node in vivo. Semin Immunol 1999; 11:73-83.

4. Butcher EC. Leukocyte-endothelial cell recognition: three (or more) steps to specificity and diversity. Cell 1991; 67: 1033-6.

5. Springer TA. Traffic signals for lymphocyte recirculation and leukocyte emigration: the multistep paradigm. Cell 1994; $76: 301$ 14.

6. Arbones ML, Ord DC, Ley K, et al. Lymphocyte homing and leukocyte rolling and migration are impaired in L- selectin-deficient mice. Immunity $1994 ; 1$ : 247-60.

7. Bistrup A, Bhakta S, Lee JK, et al. Sulfotransferases of two specificities function in the reconstitution of high endothelial cell ligands for L-selectin. J Cell Biol 1999; 145 : 899-910.

8. Maly $P$, Thall A, Petryniak B, et al. The alpha(1,3)fucosyltransferase Fuc-TVII controls leukocyte trafficking through an essential role in $\mathrm{L}-, \mathrm{E}-$, and $\mathrm{P}$-selectin ligand biosynthesis. Cell 1996; 86: 643-53.

9. Williams MB, Butcher EC. Homing of naive and memory $T$ lymphocyte subsets to Peyer's patches, lymph nodes, and spleen. J Immunol 1997; 159: 1746-52.

10. Alon R, Kassner PD, Carr MW, Finger EB, Hemler ME, Springer TA. The integrin VLA-4 supports tethering and rolling in flow on VCAM-1. I Cell Biol 1995; 128: 124353.

11. Berlin C, Berg EL, Briskin MJ et al. Alpha 4 beta 7 integrin mediates lymphocyte binding to the mucosal vascular addressin MAdCAM-1. Cell 1993; 74: 185-95.

12. Wagner, N., Lohler J, Kunkel EJ, et al. Critical role for beta7 integrins in formation of the gut-associated lymphoid tissue. Nature 1996; 382: 366-70.

13. Rott LS, Rose JR, Bass D, Williams MB, Greenberg HB, Butcher EC. Expression of mucosal homing receptor alpha4beta7 by circulating $\mathrm{CD}^{+}{ }^{+}$cells with memory for intestinal rotavirus. J Clin Invest 1997; 100: 1204-8.

14. Picker LJ, Michie SA, Rott LS, Butcher EC. A unique phenotype of skin-associated lymphocytes in humans. Preferential expression of the HECA-452 epitope by benign and malignant T cells at cutaneous sites. Am IPathol 1990; 136: 1053-68.

15. Fuhlbrigge RC, Kieffer JD, Armerding D, Kupper TS. Cutaneous lymphocyte antigen is a specialized form of PSGL-1 expressed on skin-homing T cells. Nature 1997; 389: 978-81. 
RÉFÉRENCES

16. Picker LJ, Kishimoto TK, Smith CW, Warnock RA Butcher EC. ELAM-1 is an adhesion molecule for skin-homing $T$ cells. Nature 1991 ; 349: 796-9.

17. Laudanna C, Campbell JJ, Butcher EC Role of Rho in chemoattractant-activated leukocyte adhesion through integrins. Science $1996 ; 271: 981-3$

18. Berlin-Rufenach $C$, Otto F, Mathies $M$ et al. Lymphocyte migration in lymphocyte function-associated antigen (LFA)-1- deficient mice. J Exp Med 1999; 189: 1467-78.

19. Warnock RA, Askari S, Butcher EC, von Andrian UH. Molecular mechanisms of lymphocyte homing to peripheral lymph nodes. J Exp Med 1998; 187 : 205-16.

20. Berlin C, Bargatze RF, Campbell f, et al. alpha 4 integrins mediate lymphocyte attachment and rolling under physiologic flow. Cell 1995; 80: 413-22.

21. Zlotnik A, Yoshie O. Chemokines: a new classification system and their role in immunity. Immunity $2000 ; 12: 121-7$.

22. Baggiolini $M$. Chemokines and leukocyte traffic. Nature $1998 ; 392: 565-8$.

23. Gunn MD, Kyuwa S, Tam C, et al. Mice lacking expression of secondary lymphoid organ chemokine have defects in lymphocyte homing and dendritic cell localization. J Exp Med P999; 189: 451-60.

24. Campbell Jj, Hedrick J, Zlotnik A, Siani MA, Thompson DA, Butcher EC. Chemokines and the arrest of lymphocytes rolling under flow conditions. Science 1998; 279 . 381-4.

25. Middleton I, Neil S, Wintle J, et al. Transcytosis and surface presentation of IL-8 by venular endothelial cells. Cell 1997; 91 385-95.
26. Tanaka $\mathrm{Y}$, Adams $\mathrm{DH}$, Hubscher $\mathrm{S}$, Hirano $H$, Siebenlist U, Shaw S. T-cell adhesion induced by proteoglycan-immobilized cytokine MIP-1 beta. Nature 1993; 361: 7982.

27. Sallusto F, Lenig D, Forster R, Lipp $M$ Lanzavecchia $A$. Two subsets of memory $T$ ymphocytes with distinct homing potentials and effector functions. Nature 1999; 401 . 708-12.

28. Campbell JJ, Haraldsen G, Pan J, et al The chemokine receptor CCR4 in vascular recognition by cutaneous but not intestinal memory T cells. Nature 1999; 400: 776-80.

29. Forster R, Schubel A, Breitfeld D, et al. CCR7 coordinates the primary immune response by establishing functional microenvironments in secondary lymphoid organs. Cell 1999; 99:23-33.

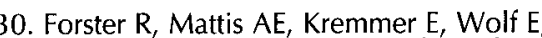
Brem G, Lipp M. A putative chemokine receptor BLR1, directs $B$ cell migration to defined lymphoid organs and specific anatomic compartments of the spleen. Cell 1996. $87: 1037-47$

31. Westermann J, Pabst R. Lymphocyte subsets in the blood: a diagnostic window on the lymphoid system? Immunol Today $1990 ; 11: 406-10$

32. Rosenberg YJ, Janossy G. The importance of lymphocyte trafficking in regulating blood lymphocyte levels during HIV and SIV infections. Semin Immunol 1999; 11: 139 54.

33. Rosenberg YJ, Anderson AO, Pabst R. HIV-induced decline in blood CD4/CD8 ratios: viral killing or altered lymphocyte trafficking? Immunol Today 1998; 19: 10-7.

34. Jacob J, Baltimore, D. Modelling T-cell memory by genetic marking of memory $T$ cells in vivo. Nature 1999; 399: 593-7.

\section{mS2000 \\ Summary}

Homing and migration: the endless journey of lymphocytes

The immune system consists of a complex collection of leukocytes and dendritic cells that surveys most tissues in the body for appearance of foreign antigens. For an efficient immune response, the interaction and co-localization of antigen-presenting cells, costimulatory helper cells and effector cells are crucial parameters. Therefore the migration routes of antigen presenting cells and of lymphocytes merge in secondary lymphoid organs in order to increase the likelihood and speed of a lymphocyte, finding its cognate antigen. Additionally, antigen primed effector cells are directed to the tissue where they are most likely to encounter their cognate antigen. This highly organized and efficient antigen encounter is based on continuous re-circulation of lymphocytes between blood, peripheral tissues and secondary lymphoid organs. Moreover, the efficacy of the immune system is further increased by the ability of different lymphocyte subsets to recirculate through distinct tissues. The scope of this review is to outline the concept and mechanisms of lymphocyte homing by underlying the molecular function of implicated cell adhesion molecules, chemokines and chemokine receptors, and to propose mechanisms by which homing physiology regulates the homeostasis of immunological resources.

\section{SOCIÉTÉ DE BIOLOGIE}

\section{L'épithélium pigmentaire de la rétine: de la cellule souche à la thérapie génique \\ Coordonnateur: Professeur Yves Courtois}

\section{Mercredi 20 septembre 2000, 16 heures}

Institut des Cordeliers,

15-21, rue de l'École-de-Médecine, 75006 Paris, France

Renseignements : Secrétariat de la Société de Biologie, Collège de France, 3, rue d’Ulm, 75231 Paris Cedex 05, France, Tél./Fax : 0144271340 